\title{
Akademische Qualitätssicherung der Komplementärmedizin: Zwischen Reglementierung und Selbstregulation
}

\author{
Hartmut Schröder \\ Institut für transkulturelle Gesundheitswissenschaften, Europa-Universität Viadrina, Frankfurt/O., Deutschland
}

Die akademische Qualitätssicherung der Komplementärmedizin durch Hochschulen findet in den letzten Jahren zunehmend durch die Einrichtung von Stiftungsprofessuren statt, die durch Lehre und Forschung wichtige Impulse geben. Der erste universitäre Studiengang für Komplementäre Medizin in Deutschland, der an der Europa-Universität Viadrina in Frankfurt/O. an einer Kulturwissenschaftlichen Fakultät etabliert wurde, stützt sich ebenfalls auf Stiftungsprofessuren und wendet sich als weiterbildender Studiengang ausschließlich an Ärzte, Apotheker, Psychologen sowie Absolventen anderer gesundheitswissenschaftlicher Fächer. In diesem Zusammenhang werden einige Probleme sichtbar: (1) Umfangreichere Lehrangebote finden vorwiegend außerhalb der medizinischen Fakultäten statt, (2) Nachhaltigkeit über mehr als 5 Jahre ist in den allermeisten Fällen nicht gesichert, und (3) Angebote akademischer Qualitätssicherung für die Vielzahl der neuen Berufe im Gesundheitswesen sind nach wie vor ein Desiderat.

Die in Berlin gegründete Academic School of Health nimmt sich dieser Probleme an und hat sich als private Hochschuleinrichtung zum Ziel gesetzt, den Gesamtbereich der Komplementärmedizin zu akademisieren und deren Qualitätssicherung nachhaltig zu garantieren. Dabei wird sie sich in Forschung und Lehre - in Kooperation mit Partnerinstitutionen aus Wissenschaft und Praxis - bedarfsorientiert Fragen rund um das Thema Gesundheit annehmen und insbesondere weiterbildende und berufsorientierte Studiengänge für die Praxis aufbauen. Ausgangspunkt und Kern der Gründung sind Wissenschaftlerinnen und Wissenschaftler des Instituts für transkulturelle Gesundheitswissenschaften der Europa-Universität Viadrina, des Institute of Complementary Medicine der Steinbeis Hochschule Berlin, der Charité Universitätsmedizin, des ZAGG - Zentrum für angewandte Gesundheitsförderung und Gesundheitswissenschaften (Berlin), des SlowMedicine Research Institute (Potsdam) und der Oberbergkliniken sowie weiterer Kliniken im Berlin-Brandenburger Raum. Unterstützt wird die neue Hochschule durch den Verband Physikalische Therapie, das Deutsche Register Osteopathischer Medizin, den Berufsverband für Atempädagogik und Atemtherapie, den Feldenkrais-Verband Deutschland, die Internationale Kinesiologie Akademie sowie zahlreiche weitere Institutionen und Verbände bis hin zu Krankenkassen.

\section{KARGER}

Fax +497614520714

Information@Karger.de

www.karger.com (c) 2010 S. Karger GmbH, Freiburg

Accessible online at:

www.karger.com/fok
Durch die Gründung der neuen Hochschule werden auch Vorgaben der Weltgesundheitsorganisation und des Gesetzgebers in Deutschland erfüllt. So hat die WHO in ihrer «Bejing Declaration», die von der 61. Versammlung der Weltgesundheitsorganisation als verbindlich beschlossen wurde, mit deutlichen Worten darauf hingewiesen, dass natürliche und traditionelle Medizinsysteme bevorzugt beforscht, auf ihre Nützlichkeit hin untersucht sowie, wo sinnvoll, in die medizinische Versorgung der Mitgliedsländer integriert werden sollen. In $\S 2$ des deutschen Sozialgesetzbuches V wurde der Begriff der «besonderen Therapierichtungen» bereits 1989 eingeführt; wörtlich heißt es in Absatz 1: «Behandlungsmethoden, Arznei- und Heilmittel der besonderen Therapierichtungen sind nicht ausgeschlossen. Qualität und Wirksamkeit der Leistungen haben dem allgemein anerkannten Stand der medizinischen Erkenntnisse zu entsprechen und den medizinischen Fortschritt zu berücksichtigen» sowie weiter in Absatz 3: «Bei der Auswahl der Leistungserbringer ist ihre Vielfalt zu beachten.»

Ernst Boxberg, Fachanwalt für Medizinrecht und einer der Gründer der neuen Hochschule, weist darauf hin, dass der Gesetzgeber als besondere Therapierichtungen zwar ausdrücklich homöopathische, phytotherapeutische und anthroposophische Arznei- und Heilmittel nennt, aber - wie die Ausschussbegründung zu diesen gesetzlichen Tatbeständen zeigt - an die Naturheilkunde im weiteren Sinne gedacht hat, sich also noch nicht wirklich festgelegt hat, was konkreter Inhalt der komplementärmedizinischen Elemente sein soll. Boxberg zählt zur Komplementärmedizin fünf große Bereiche: (1) Mind-Body-Medicine als Erweiterung der geistigen Fähigkeit zur Beeinflussung körperlicher Funktionen und Symptome durch Meditation, mentales Heilen und künstlerische Methoden; (2) biologisch fundierte Methoden (natürliche Methoden wie Kräuter, Nahrungsmittel und Vitamine); (3) manipulative körperbezogene Methoden (bei denen Körperteile manipuliert oder bewegt werden, wie in der Massage, der Osteopathie oder der Chiropraktik); (4) energetische Methoden (die Biofeldtherapien und bioelektromagnetische Therapien anwenden); (5) ganze Medizinsysteme (Homöopathie und Anthroposophie sowie die Systeme der traditionellen chinesischen Medizin oder die ayurvedische Medizin). 
In all diesen Bereichen - so Boxberg - sind seit Jahrzehnten Berufe außerhalb der medizinischen Fakultäten entstanden, die weder vom Gesetzgeber erfasst noch reglementiert werden. Dadurch wird auch erfolgreichen Heilkundigen die Betätigungsmöglichkeit vorenthalten oder erschwert. Die Gründung der neuen Hochschuleinrichtung setzt hier an und reflektiert einerseits die Veränderungsprozesse im Gesundheitssystem sowie andererseits in den Gesundheitswissenschaften und in der Medizin selbst, wo sich trotz der weiter bestehenden Dominanz der Naturwissenschaften in den letzten Jahren mit dem beginnenden $\mathrm{Pa}$ radigmenwechsel von einem biomedizinischen zu einem biopsychosozialen Verständnisansatz zugleich eine «kommunikative Wende» vollzogen hat, durch die die Medizin wieder stärker mit ihren geisteswissenschaftlichen Wurzeln verbunden wird und sich ihrer Stellung zwischen Wissenschaft und Kunst zurückbesinnt.

Ein weiterer Paradigmenwechsel zeigt sich im Gesundheitssystem, wo auf der Grundlage sich wandelnder Krankheitsmuster, zunehmender Bedeutung von Prävention, Gesundheitserziehung und Gesundheitskommunikation Begriffe wie «psychosoziale Gesundheit» und «Lebensqualität» eine immer größere Rolle spielen. Dieser Prozess verlangt eine ganzheitliche Heilkunst und hat bereits in einer Vielzahl neuer Studiengänge Ausdruck gefunden. In einem Grundsatzpapier des «Health Capital Netzwerkes» (Gesundheitswirtschaft Berlin-Brandenburg) schreibt Günter Stock, der Präsident der Berlin-Brandenburgischen Akademie der Wissenschaften: «Gesundheit ist mehr als das Gegenteil von Krankheit, und «Studium Gesundheit 〉 bedeutet mehr als «Medizinstudium〉. Es geht bei diesem Thema heute um Lebensqualität im weitesten Sinne: um Medizininformatik ebenso wie um biochemische Alzheimerforschung, um Rehabilitation ebenso wie um Gesundheitserziehung, um Pharmaforschung oder Pflegemanagement ebenso wie um Neurowissenschaften, Ernährung oder Gesundheitstourismus.»

Der Paradigmenwechsel im Gesundheitssystem drückt sich bereits in der «Gesundheitswirtschaft» aus, wobei sich eine besondere Dynamik im «Zweiten Gesundheitsmarkt» feststellen lässt, zu dem die privat finanzierten Produkte und Gesundheitsleistungen gehören. Geht es im «Ersten Gesundheitsmarkt» um die medizinische Normalversorgung auf der Basis des alten mechanistischen Modells von Krankheit, so steht im Zweiten Gesundheitsmarkt die Salutogenese im Mittelpunkt. Der Zweite Gesundheitsmarkt erfreut sich im Gegensatz zum Ersten Gesundheitsmarkt eines außerordentlich positiven Images, er entlastet den Ersten Gesundheitsmarkt durch die Orientierung auf Prävention und trägt insgesamt positiv zur Wertschöpfung der Gesundheitswirtschaft bei. In Deutschland hat der Zweite Gesundheitsmarkt bereits ein Volumen von über 60 Milliarden Euro erreicht, wobei die Nachfrage größer als das Angebot ist.

Die meisten komplementärmedizinischen Angebote gehören in Deutschland noch immer zu den «Individuellen Gesundheitsleistungen» (IGel), d.h. zu den medizinisch im Ersten Gesundheitsmarkt als nicht notwendig angesehenen Leistungen, die individuell zu bezahlen sind. Dass dieser Bereich sowohl von den Patienten gewünscht wird als auch auf eine entsprechende Kaufkraft stößt, zeigen verschiedene Befragungen. Danach wünschen Patienten sich mehr Differenzierung und über $90 \%$ geben an, dass sie durchaus vom Standardangebot abweichende Tarife mit zusätzlichen Leistungen wählen würden. Die Gesetzlichen Krankenkassen haben diese Entwicklung mittlerweile erkannt und stellen sich auf die neue Situation ein, indem über Wahltarife auch Produkte und Leistungen der besonderen Therapierichtungen durch Kassenpatienten in Anspruch genommen werden können. Auf diese Weise verschmelzen der erste und der zweite Gesundheitsmarkt zunehmend miteinander und Forderungen nach mehr Transparenz, Vergleichbarkeit und Qualitätssicherung der komplementären Angebote werden von den Akteuren geäußert.

In diesem Umbruchprozess wird eine vernünftige Form interner Qualitätssicherung mit darüber entscheiden, ob sich die insgesamt positive Entwicklung der komplementären Medizin weiter fortsetzt und eine Integration in die Normalversorgung durchgesetzt werden kann oder ob sie durch Versuche der Regulierung und Reglementierung von außen gehemmt wird. Der Zweite Gesundheitsmarkt blieb zwar bislang weitgehend von einer gesetzlichen Verpflichtung der externen Qualitätssicherung verschont, als Preis dafür bleibt er aber für die Patienten in vielen Bereichen undurchsichtig und für die Kassen und Versicherungen hinsichtlich erstattungsfähiger Leistungen nur schwer zu überblicken.

Angesichts der zunehmenden Bedeutung des Zweiten Gesundheitsmarktes kann damit gerechnet werden, dass es über kurz oder lang Versuche der Akteure der Gesundheitswirtschaft geben wird, eine Reglementierung dieses Bereichs von oben nach unten durchzusetzen. Diese Reglementierung wird dabei umso tiefgreifender sein, je unregulierter die einzelnen Segmente erscheinen. Eine überzeugende interne Qualitätssicherung, die von Akteuren freiwillig, eigenständig und selbstverantwortlich betrieben wird, dürfte daher strategisch außerordentlich wichtig sein, um Qualitätskriterien und Qualitätsmanagementsysteme mitgestalten zu können. Die akademische Absicherung, d.h. die Einrichtung von sowohl grundständigen als auch weiterbildenden Studiengängen für die Vielzahl der neuen Berufe und Qualifikationen in der Gesundheitswirtschaft, ist in der gegenwärtigen Umbruchphase von allergrößter Bedeutung. Gefragt sind in dieser Hinsicht aber nicht nur innovative Studienangebote, sondern vor allem auch die Verknüpfung von Lehre und Forschung auf einem hohen Niveau. Nur durch exzellente Forschung kann die Akzeptanz der komplementären Medizin und der neuen Berufe weiter gesichert werden.

Mit diesen Aufgaben scheinen staatliche Hochschulen aber eher überfordert zu sein, da sie sich nur ungenügend auf die Dynamik des Zweiten Gesundheitsmarktes und die neuen Gestaltungsmöglichkeiten vorbereitet haben bzw. nur langsam auf diese Entwicklungen überhaupt reagieren (können). Die Gründung einer eigenständigen und privaten Hochschuleinrichtung stand damit auf der Tagesordnung. Ihr Erfolg wird nun nicht zuletzt davon abhängen, ob sie dem weiten Feld der Komplementärmedizin eine akademische Heimat geben kann, ohne damit einen Richtungsanspruch zu verbinden. Gelingen wird dies, wenn sie allen Beteiligten so wenig wie möglich Struktur und Regulierung vorgibt und so viel wie möglich Raum zur Entwicklung und Selbstregulation lässt. Ellis Huber - ehemaliger Präsident der Berliner Ärztekammer und einer der Gründer der neuen Hochschule - hat die selbstgesetzte Zielsetzung in der «TOP-Formel» zum Ausdruck gebracht: Tolerant - offen - pluralistisch! 